\title{
Das Berliner Ostrakon P. 14735: Koptisch oder Griechisch?
}

\author{
Mit Abbildung 4
}

Klaas A. Worp (Amsterdam)*)

Die ed. princeps des Berliner Ostrakons P.14735 findet sich in ZÄS 104 (1977) 110-112 (mit Taf. 18 und 19), wo der Text, zusammen mit einigen anderen in Elephantine gefundenen koptischen Ostraka, so gelesen wurde:

\author{
P егW MAKAPIOC AlOY XPEWCT1 \\ Gn חPAIחOCITW nNAYAON

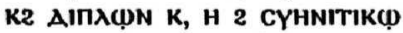

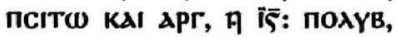 \\ 5 TI, noגy $\cap$ is $=$ \\ CYHN, $\boldsymbol{K}, \boldsymbol{H} ?=$ \\ фАWф1 r THC IE INA, \\ A) $\mathrm{GM} \overline{\mathrm{O}}$ сגх, егрАфн \\ (Rs.) 0 xol, 月 $a, \eta \pi \circ \lambda \beta$,
}

Der Herausgeber F. Hintze bemerkt zu seinem Text: „Sprachlich liegt eine merkwürdige Mischung von Griechisch und Koptisch vor. Formal handelt es sich um einen Schuldschein, bei dem nicht alle Einzelheiten klar sind. ... Da die Schuldsumme ziemlich hoch ist (16 Unzen [ $=96$ Solidi] und $8 \frac{1}{2}$ Karat), handelt es sich offensichtlich nicht um private Steuerschulden des Makarios, sondern um dessen Verpflichtung, die betreffenden Steuern für seinen Steuerbezirk einzutreiben. Die Verpflichtung in Form eines Schuldscheines deutet darauf hin, daß der Steuereintreiber für die Steuersumme persönlich mit seinem Vermögen haftete." Nach Hintzes Ansicht handelt es sich bei den Schul-

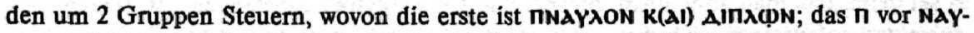
$\lambda O N$ sollte wohl nicht als der koptische Artikel, sondern vielleicht als (Y)n(EP) aufgelöst werden. Die zweite Steuergruppe besteht seiner Meinung nach aus ncrTW KAI Apr, der Betrag ist 16 Unzen, und man sollte wohl auflösen als (Y)n(EP) CITOY KAI Apr(YPIKCN).

Obwohl gegen eine solche Betrachtungsweise vielleicht zunächst wenig zu sprechen scheint, erheben sich doch Probleme: Man erwartet in einem solchen Text kein Zusammengehen von Getreide und Geldsteuern zu dem Betrag von 16 Unzen; die Kombination von Keratien mit dem Wort cүнnrrıк $\omega$ in $\mathrm{Z} .3$ läßt vermuten, daß die Münzen hier,mit

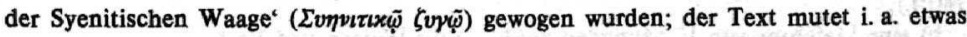

*) Gern danke ich Prof.Dr. D.Hagedorn und Prof. Dr. P.J. Sijpesteijn, mit denen ich das Ostrakon diskutieren konnte und die mir wichtige Informationen besorgt haben. Dr. G. Poethke beschaffte nicht nur ein schönes Photo der Vorderseite, sondern prüfte auf meine Bitte einige Stellen am Original und mein Deutsch, wofür ich ihm besonders dankbar bin. 
mehr griechisch als koptisch an (im Koptischen würde man wohl eher dNOK statt ero geschrieben haben) und sollte dann auch im Prinzip mit griechischen Buchstaben gedruckt werden. Die Tafel der Vorderseite zeigt, daß zwar die Schrift hier nicht unbedingt als Griechisch zu betrachten ist; eine ähnliche Schrift aber begegnet auch in anderen griechisch geschriebenen Ostraka aus dem byzantinischen Ägypten (die Rückseite ist sowieso griechisch geschrieben). Auch ergab die Überprüfung, daß an einigen Stellen vielleicht an eine andere Lesung gedacht werden kann. Auf Grund eines vorzüglichen Photos, das Dr. G. Poethke, Kustos der Berliner Papyrussammlung, mir besorgte, möchte ich folgende Neulesung des Ostrakons vorschlagen:

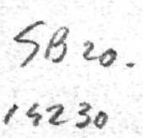

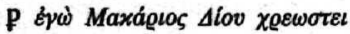

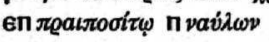

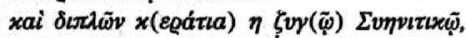

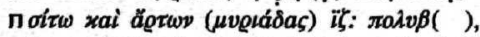

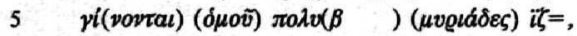

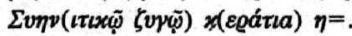

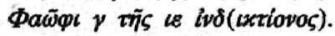

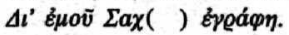

(Rs.) $\delta \mu o i(\omega \zeta)(\mu v \varrho \iota a ́ \delta a) a, \eta \pi \sigma d v \beta(\quad)$.

\section{$1 \chi \varrho \varepsilon \omega \sigma \tau \tilde{\omega}$ ? $3 \zeta v y$ über $\sigma v \eta \quad 4 \sigma t \tau o v$}

"Ich, Makarios, Sohn des Dios, schulde dem praepositus für naula und dipla $8 \mathrm{~K}$ (eratien) mit der Syenitischen Waage (gewogen) (und) für Weizen und Brot 17 Myriaden polyb( ), macht zusammen polyb( ) 17 Myriaden, mit der Syenitischen Waage $8 \mathrm{~K}$ (eratien). Am 3.Phaophi der 15. Indiktion. Von mir, Sach( ), geschrieben. (Rs.) Ebenfalls 1 Myriade, 8000 polyb( ). .

Hieraus erhellt, daß ich den Text als Griechisch, wenn auch mit koptischen Elementen,

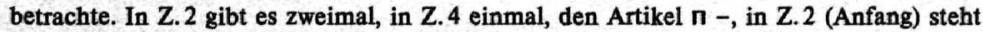
die Präposition e - (+ Dativ = ,an, nach', vgl. W. E. Crum, Coptic Dictionary, 50). Wie schon erwähnt, entspricht E $\gamma \omega \dot{\omega}$ hier dem ANOK koptischer Quittungen. Mit der Verbalform $\chi \varrho \varepsilon \omega \sigma \tau \varepsilon$ gibt es insoweit Schwierigkeiten, daß man im Griechischen eine 1.Ps. Sing. braucht (1. $\chi \varrho e \omega \sigma \tau \tilde{)})$, die Kopten die griechischen Verba vielfach in der Form der 2.Ps.Sg.Imperativ übernommen haben (vgl. W. C. Till, Koptische Dialektgrammatik [1961] 40 § 187). Man kann die Form $\chi \varrho e w o t \varepsilon \imath$ also als einen Koptizismus betrachten ( $\chi \varrho \varepsilon \omega \sigma \tau \varepsilon t)$, es sei denn, da $B$ man annehmen möchte, da $B$ der Schreiber angefangen hat, seinen Text ,subjektiv' aufzusetzen, sich dann aber geirrt hat und in ,objektivem' Stil weitergefahren ist (xeewarei).

Tatsächlich betrachte ich diesen Text als einen Schuldschein, bei dem zwei Bestandteile zu unterscheiden sind:

a) eine Zahlung für naula (Transportkosten) und dipla (= Verpflegungskosten? Vgl. O. Cair. GPW 71, 6 Anm.) zu 8 Keratien ,mit der Syenitischen Waage gewogen' (vgl. P. Monac. I 1, 53 Anm.; L. C. West-A. C. Johnson, Currency in Roman and Byzantine Egypt $140 \mathrm{ff}$ ),

b) eine Lieferung von 17 Myriaden polyb ( ) für Weizen und Brot.

Weil unter a) von Geld die Rede ist, scheint es logisch, auch unter b) an Geld zu denken. Tatsächlich gab es eine Münze, Myriade' (vgl. R. S. Bagnall, Currency and Inflation in Byzantine Egypt 12). Wenn dies für den vorliegenden Text zutrifft, würden die Myriaden mit der näheren Qualifikation, polyb( $)^{c}$, deren Bedeutung (es könnte sich um ein Adjek- 
tiv handeln) unbekannt ist, definiert worden sein. Man kann aber auch davon ausgehen, daß es sich hier nicht um Myriaden als Münze, sondern einfach um die Zahl ,10000‘ handelt. In diesem Falle wären 170000 ,polyb( )‘ [ein Substantiv unbekannter Bedeutung] geliefert worden. Hierfür kann auch die Rückseite sprechen, wo neben der einen Myriade die Zahl ,8000' ohne Hinweis, worauf sich diese Beträge beziehen, erscheint. Zwar kommt es vor, daß ein Drachmen-Siglum in einer Zusammensetzung mit Talenten fehit. Hier aber könnte man daran denken, daß „ebenfalls 1 Myriade, 8000 [macht zusammen $18000], p o l y b()^{c u}$ geliefert wurden. Jedenfalls sollte das Siglum $\sqcap$ nicht als Unze, sondern als Myriade betrachtet werden. Unzen werden in den Papyri und Ostraka üblicherweise mit dem Symbol $\mathrm{F}$ bezeichnet, während eine Art Bogen mit einem Punkt in der Mitte das übliche Siglum für $10000^{6}$ (= 1 Myriade) ist. ${ }^{1}$ )

In der ed. princ. wird zu Z. 8 vorgeschlagen, den Schreibernamen CAx(Aplac) zu lesen, was am wahrscheinlichsten ist. Es läßt sich auch an andere Möglichkeiten denken; allerdings scheint die Auflösung $\Sigma a \chi(o \mu v \varepsilon ́ \omega \varsigma$ ) weniger wahrscheinlich, weil der Name bis jetzt vorzugsweise in thebanischen Ostraka begegnet.

Um welche Art praepositus es sich hier in Z. 2 handelt, ist nicht klar; im Zusammenhang mit dem Fundort des Ostrakons, Elephantine, denkt man selbstverständlich an den militärischen praepositus limitis (vgl. J. Maspero, L'organisation de l'Egypte byzantine, 101-3), aber auch andere Funktionäre sind denkbar (vgl. F. Preisigke, WB III, Abschn. 8,

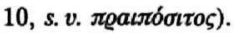

Abschließend sollte bemerkt werden, daß auch in anderen griechischen Ostraka vom praepositus oder dessen Gehilfen die Rede ist, die Waren entgegenzunehmen; vgl. z. B. O.Leid. 345, O. Bodl. II 2111, 2117, 2119 und 2098, 2099. Die meisten dieser Texte, deren Herkunft nicht feststeht, sind von ihren Herausgebern auf das IV. oder V.Jh. datiert worden, nur O. Bodl. II 2117 wird auf das VI.(?) Jh. datiert. Auch für unseren Text scheint eine paläographische Datierung der Schrift auf das VI. oder sogar das VI./VII. Jh. möglich. Einige Ostraka, die zum Archiv des Theopemptos und Zacharias gehören (zu dessen Datierung vgl. P. Oxy. LV 3797,9 Anm.; auch O. Bodl. II 2117 dürfte zu diesem Archiv gehören), weisen eine ähnliche Schrift auf. Ein spätes Datum für dieses Ostrakon erklärt um so leichter das Zusammengehen von griechischen und koptischen Elementen in diesem interessanten Text.

1) Vg1. W. M. Brashear in ZPE 60 (1985) 239-242. 